\title{
Analysis of the Relative Motion Between the Socket and Residual Limb in Transtibial Amputees While Wearing a Transverse Rotation Adapter
}

\author{
Corey A. Pew, ${ }^{1}$ Sarah A. Roelker, ${ }^{2}$ Glenn K. Klute,,${ }^{3,4}$ and Richard R. Neptune ${ }^{2}$ \\ ${ }^{1}$ Montana State University; ${ }^{2}$ The University of Texas at Austin; ${ }^{3}$ University of Washington; ${ }^{4}$ Center for Limb Loss and Mobility
}

\begin{abstract}
The coupling between the residual limb and the lower-limb prosthesis is not rigid. As a result, external loading produces movement between the prosthesis and residual limb that can lead to undesirable soft-tissue shear stresses. As these stresses are difficult to measure, limb loading is commonly used as a surrogate. However, the relationship between limb loading and the displacements responsible for those stresses remains unknown. To better understand the limb motion within the socket, an inverse kinematic analysis was performed to estimate the motion between the socket and tibia for 10 individuals with a transtibial amputation performing walking and turning activities at 3 different speeds. The authors estimated the rotational stiffness of the limb-socket body to quantify the limb properties when coupled with the socket and highlight how this approach could help inform prosthetic prescriptions. Results showed that peak transverse displacement had a significant, linear relationship with peak transverse loading. Stiffness of the limb-socket body varied significantly between individuals, activities (walking and turning), and speeds. These results suggest that transverse limb loading can serve as a surrogate for residual-limb shear stress and that the setup of a prosthesis could be individually tailored using standard motion capture and inverse kinematic analyses.
\end{abstract}

Keywords: inverse kinematics, simulation, prosthesis, below-knee amputee

The coupling of lower-limb prosthetic components to the individual's residual limb presents many challenges. Often, the high loads of ambulation are transmitted through the soft tissues to the skeletal system, which can produce damaging shear stresses. ${ }^{1-3}$ Shear stress can be particularly high during turning and twisting activities as most prosthetic legs allow limited motion in the transverse plane ${ }^{4}$ which requires the residual limb to absorb the majority of the displacement. Transverse rotation adapters (TRAs) have been shown to absorb some of this motion and reduce loading on the residual limb. ${ }^{5-7}$ The use of a TRA has also been shown to reduce metabolic cost and step width during straight-line walking, ${ }^{8,9}$ improve turning and walking over rough terrain, ${ }^{9}$ and marginally increase activity level. ${ }^{10}$ In addition, our recent studies using a variable-stiffness TRA have shown that lower adaptor stiffness values can significantly reduce loading at the socket in the transverse plane ${ }^{1-14}$ and that different stiffness settings may be beneficial for varying activities and walking speeds compared with current single-stiffness TRAs. While reductions in loading are generally regarded as beneficial, there is no established practice to determine the proper stiffness of a TRA that will provide the greatest user benefit when balancing comfort, mobility, and user preference. ${ }^{14}$ Currently, the setup of a TRA is adjusted iteratively using the subjective experience of the prosthetist and feedback from the user. While this process

Pew is with the Department of Mechanical and Industrial Engineering, Montana State University, Bozeman, MT, USA. Roelker and Neptune are with the Walker Department of Mechanical Engineering, The University of Texas at Austin, Austin, TX, USA. Klute is with the Department of Mechanical Engineering, University of Washington, Seattle, WA, USA; and the Department of Veterans Affairs, Center for Limb Loss and Mobility, Seattle, WA, USA. Pew (Corey.Pew@montana. edu) is corresponding author. generally results in an acceptable setup, a more objective method to determine the optimal stiffness setting could increase efficiency and consistency across individuals.

One main drawback of previous research aimed at characterizing the influence of a TRA on residual limb loading is the difficulty in measuring soft-tissue shear stress directly. In general, reductions in loading should relate to reductions in displacement between the socket and residual limb that cause shear stress. However, no analysis has been performed to directly relate these 2 quantities. Motion of the residual limb is difficult to measure experimentally via motion capture as markers cannot be placed on the residual limb inside of a conventional socket. ${ }^{15}$ While some experimental methods have been developed to help estimate, visualize, and measure this movement, ${ }^{16-19}$ these methods only quantify motion between the socket and socket liner surface and do not quantify motion of the deeper soft tissues. This is important as shear of the skin and muscle tissues can result in deep tissue injury in lower-limb amputees, which can also lead to muscle lesions and occlusion of blood flow. ${ }^{2,20}$ Some studies have utilized imaging techniques such as X-ray and ultrasound to image the motion of the skeletal system relative to the socket. ${ }^{21-25}$ However, these methods are limited to images taken during static poses, they expose individuals to harmful radiation, and often require the creation of custom sockets to house imaging equipment.

Models of the lower limbs as mechanical systems have been developed to understand how devices such as a TRA interact with the body and aid in the design and setup of prosthetic devices. ${ }^{26-31}$ These studies have generally focused on the mechanics of the foot and ankle with little attention paid to the socket-residual limb interface. Using finite element analyses, others have sought to understand the mechanical properties, shear stresses and strains, and displacements being experienced by the soft tissues inside of a 
lower-limb prosthesis. ${ }^{2,19,32,33}$ However, they are limited by using soft tissue properties from a small number of individuals that are taken statically instead of during dynamic gait events. While these studies have provided useful information about how the limb may behave mechanically during standing and the interactions that may occur inside of the socket, they do not provide estimates during ambulation or the ability to easily customize that analysis for a specific individual. This is because the specific tissue composition, the location of the bony anatomy within the residual limb, and the specific interface between the socket and residual limb can vary widely across individuals, making it difficult to quantify the motion between the socket and residual limb.

Methods utilizing modeling and simulation to estimate the motions between the prosthetic socket and residual limb using inverse kinematic (IK) approaches have also been developed to help understand the motions of the residual limb inside of the socket. ${ }^{15,34}$ IK methods calculate the position of individual bodies in the model and are used to estimate the unknown motion between the socket and residual limb using data from a standard motioncapture experiment. The objective of our present study was to relate the relative rotational motion between the residual limb and socket that causes shear stresses in the soft tissues and measured transverse plane load applied to the distal end of the socket using an IK analysis. In addition, an estimation of the physical attributes of an individual's residual limb and how it interacts with their socket could provide a quantitative approach for determining an ideal stiffness for a TRA. To this end, we used the measured load and estimated displacement to quantify the transverse rotational stiffness of the socket-limb body (liner, skin, and soft tissues combined) for individual subjects and activities (walking and turning) performed at different speeds, which were then used to model the lower limb of the amputee and provide an individual specific setup for a TRA prescription. Specifically, we hypothesize that (1) the peak transverse plane moment will have a positive, linear relationship to the peak estimated relative motion between the socket and residual limb and (2) the transverse stiffness of the residual limbsocket body will vary based on each individual subject, activity, and speed.

\section{Methods}

Ten unilateral, transtibial amputees ( 1 female, age: mean 51 [SD 17] y, mass: 84 [13] kg, height: 1.78 [0.08] m, Table 1) gave their informed consent to this protocol reviewed and approved by the Veterans Affairs Puget Sound Health Care System and the University of Washington. ${ }^{14}$ Inclusion criteria required that subjects: (1) be 18-75 years of age, (2) be a unilateral or bilateral, transtibial amputee, (3) have been fit with a prosthesis and used it for at least 6 months, (4) use the prosthesis for at least 4 hours per day by selfreport, (5) are able to ambulate without the use of extremity aids, and (6) have no concurrent musculoskeletal injuries or other health conditions that would prevent them from completing the full protocol by self-report. Gait kinematics and kinetics were collected using a 12-camera Vicon system (Vicon, Centennial, CO), 8 force plates (AMTI, Watertown, MA), and an iPecs 6-axis load cell (College Park, Warren, MI) installed on the distal end of the socket to directly measure transverse plane loading of the residual limb (normalized by body mass). Kinetic data (force plates and iPecs) were recorded at $1200 \mathrm{~Hz}$ while kinematic data were recorded at $120 \mathrm{~Hz}$. Raw data were processed using a low-pass Butterworth filter at 25 and $6 \mathrm{~Hz}$ for the kinetic and kinematic data, respectively. Subjects wore an adjustable TRA during testing using 3 fixed stiffness settings (compliant: $0.25 \mathrm{~N} \cdot \mathrm{m} / \mathrm{deg}$, intermediate: $0.75 \mathrm{~N} \cdot \mathrm{m} /$ deg, stiff: $1.25 \mathrm{~N} \cdot \mathrm{m} / \mathrm{deg}$ ) at 3 speeds (self-selected, fast, and slow: $\pm 20 \%$ of self-selected, respectively) during 3 activities (straight-line walking [ST], turning with the prosthesis on the inside of the turn [PI], and turning with the prosthesis on the outside of the turn [PO], 27 conditions total). Straight-line, steady-state walking was performed overground along a 10-m walkway, and turning was performed by walking around a 1-m radius circle. ${ }^{14,35}$ Participants wore their asprescribed socket and suspension and were fit by a certified prosthetist with the experimental TRA and a Vari-Flex Low Profile foot (Össur, Reykjavík, Iceland).

Markers were placed on the subjects by the same individuals across all subjects to improve repeatability of placement. All subjects were instrumented with 71 motion-tracking markers according to a modified Plug-in-Gait full-body model (Vicon). ${ }^{12}$ Modification of the model included the addition of marker clusters on the thighs and upper arms and markers on the first and fifth metatarsal heads, tibial tuberosity, fibula head, and the medial aspects of the ankle, knee, and elbow joints. Femoral condyle markers were placed on the prosthetic socket by asking the subject to bend their knee several times and visually aligning the markers to the axis of knee rotation. Tibial tuberosity and fibular head markers were placed on the outside of the socket on the affected side and matched to their locations on the residual limb as closely as possible by visual inspection.

\section{Table 1 Demographic Data for Participants}

\begin{tabular}{|c|c|c|c|c|c|c|c|c|}
\hline Subject & $\begin{array}{l}\text { Amputation } \\
\text { etiology }\end{array}$ & $\begin{array}{c}\text { Time since } \\
\text { amputation, y }\end{array}$ & Liner (brand, sock plies) & Age & $\begin{array}{l}\text { Height, } \\
\text { m }\end{array}$ & $\begin{array}{l}\text { Weight, } \\
\text { kg }\end{array}$ & $\begin{array}{l}\text { Straight } \\
\text { ssW, m/s }\end{array}$ & $\begin{array}{c}\text { Turn SSW, } \\
\mathrm{rad} / \mathrm{s}\end{array}$ \\
\hline 1 & Trauma & 23 & WillowWood Alpha, 3-ply & 49 & 1.79 & 85.5 & $1.21(0.02)$ & $0.84(0.03)$ \\
\hline 2 & Trauma & 6 & Össur Iceross Synergy & 26 & 1.76 & 87.5 & $1.32(0.03)$ & $1.04(0.03)$ \\
\hline 3 & Trauma & 43 & WillowWood Alpha, 5-ply & 70 & 1.83 & 87.8 & $1.23(0.04)$ & $0.60(0.03)$ \\
\hline 4 & Trauma & 8 & WillowWood Alpha & 48 & 1.87 & 98.9 & $1.38(0.03)$ & $0.99(0.04)$ \\
\hline 5 & Trauma & 49 & Össur Iceross Sport & 67 & 1.84 & 92.1 & $1.32(0.05)$ & $0.96(0.03)$ \\
\hline 6 & Trauma & 11 & Össur Iceross Comfort, 5-ply & 41 & 1.81 & 72.6 & $1.43(0.05)$ & $1.00(0.04)$ \\
\hline 7 & Tibial hemimelia & 21 & Össur Iceross Sleeve, 1-ply & 25 & 1.58 & 48.9 & $1.23(0.04)$ & $0.99(0.04)$ \\
\hline 8 & Cancer & 1 & WillowWood Alpha, 13-ply & 68 & 1.74 & 80.9 & $1.24(0.02)$ & $1.01(0.02)$ \\
\hline 9 & Diabetic/vascular & 3 & WillowWood Alpha, 3-ply & 70 & 1.75 & 91.0 & $1.18(0.03)$ & $0.74(0.04)$ \\
\hline 10 & Trauma & 2 & Össur Iceross Dermo & 42 & 1.80 & 92.5 & $1.03(0.03)$ & $0.76(0.04)$ \\
\hline
\end{tabular}

Abbreviation: SSW, self-selected walking speed. Note: Straight-line walking and turning SSWs reported for each individual, mean (SD). 
A generic musculoskeletal model for the transtibial amputees was developed based on the work of LaPré et al. ${ }^{15}$ Briefly, the Gait2354 OpenSim model ${ }^{36}$ was modified by transecting the affected tibia and removing all bodies and muscles distal to the transection point. ${ }^{15}$ As recommended by LaPré et al, ${ }^{15}$ the motion between the residual tibia and socket segments of the model were restricted to 4 degrees of freedom, 3 rotational degrees of freedom, and 1 translational degree of freedom along the length of the tibia (ie, to allow pistoning). In addition, the LaPré et al $^{15}$ model was further modified to include a rotational degree of freedom between the prosthetic socket and foot segments to accommodate the transverse plane motion of the TRA (Figure 1). In OpenSim (version 4.0), ${ }^{36}$ the model was scaled for each individual subject by minimizing the difference between the experimental location of 22 markers on the subject during a static trial and their respective virtual locations on the model, which included the $\mathrm{C} 7$ spine, clavicle, left and right anterior superior iliac spine, posterior superior iliac spine, lateral and medial femoral condyles, lateral and medial malleoli, first and fifth metatarsal heads, tip of the second toe, and heel. Because markers could not be placed at the effective center of rotation on the prosthetic ankle, the amputated tibia and prosthetic socket were scaled proportionally to the intact tibia. The IK analyses were performed via a MATLAB (MathWorks, Natick, MA) interface with OpenSim to allow batch analyses of trials from each subject during each activity and speed combination. Tracking markers for the IK analysis included all those from the scaling set and added the left and right acromion processes, tibial tuberosity, fibular head, and clusters of 4 markers on the thighs. The IK analyses solved a weighted least squares problem to

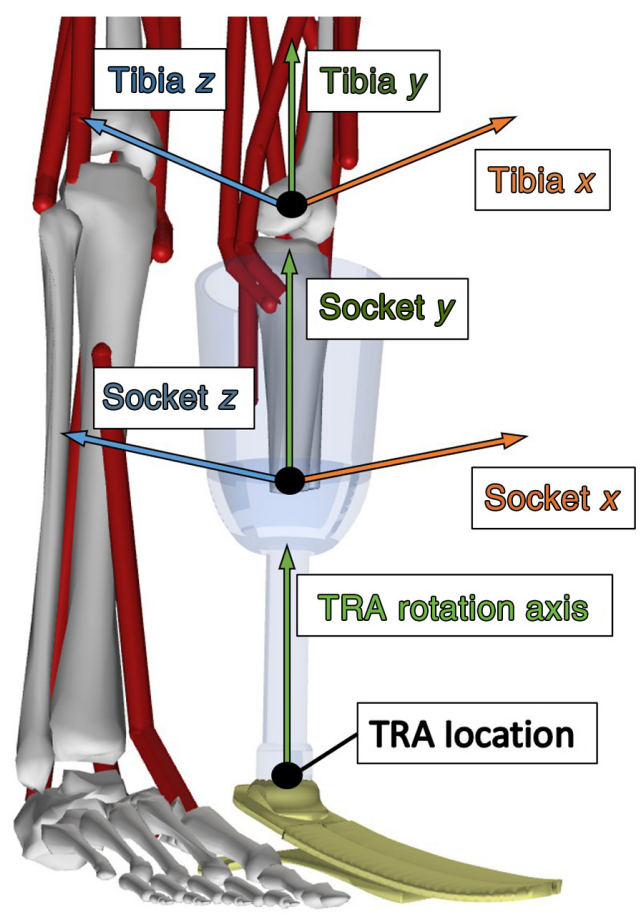

Figure 1 - Prosthetic limb model detail. Origins are defined at the proximal end of each segment. The 4-degree-of-freedom model between the socket and transected tibia allows for rotation between the tibia and socket in all 3 axes as well as translation along the $y$-axis (pistoning). An additional degree of freedom was also added to accommodate TRA rotation about the $y$-axis. TRA indicates transverse rotation adapter. minimize the error between the experimental and virtual marker locations during each activity trial. Maximum marker error during the IK analysis was limited to $<4 \mathrm{~cm}$, and any trials that were not able to meet that specification were not used for the analysis. ${ }^{37}$

The relationship between the measured peak transverse plane moment and estimated peak rotation between the socket and residual limb was analyzed using a linear regression model with fixed effects for stiffness setting, activity, speed, and subject with an intercept through the origin. The fixed intercept at the origin was used based on the assumption that at zero load, there will be zero deflection. A linear mixed-effects model with fixed effects for stiffness setting, activity, speed, and random effects for the individuals was used to investigate relationships between the TRA stiffness setting and estimated rotation. The limb-socket body stiffness was calculated by dividing the measured peak force at the socket by the peak estimated motion from the IK analysis. A repeated measures analysis of variance was used to determine if limb-socket stiffness varied between the different individuals, activities, and speeds. If an overall difference was found, pairwise comparisons were performed using the estimated marginal means and Tukey method for comparing families to determine specific differences. Data were evaluated only during the stance phase of each task when the limb was loaded. ${ }^{3}$ Comparisons were made for all activities together as well as for each activity individually, with significant relationships indicated by $P<.05$. The use of the iPecs load cell allowed for multiple-stance phase measurements during each trial (ie, multiple steady-state steps). With each of the 10 subjects performing multiple trials for the varying conditions, a total of 4060 stance phases were used for this analysis.

\section{Results}

The analysis identified a positive, linear relationship between the peak transverse plane moment and estimated relative motion between the socket and residual limb. Qualitatively, the patterns of the estimated rotation matched those of the measured transverse loading across the 3 activities during the stance phase (Figure 2). Significant, positive, linear relationships between the peak transverse plane moment and peak estimated relative rotation between the socket and residual limb were found for all activities (walking and turning; Figure 3; all $P$ s $\leq .030$ ), with the exception of external rotation during straight-line walking (Table 2). In addition, we found that the linear slope differed between internal and external rotations for all activities $(P \leq .005)$. The peak estimated rotation results showed that, on average, across TRA settings, activities and speeds of the residual limb experienced 16 (SD 13) deg of displacement relative to the socket. Straight-line walking and PI turning are both primarily composed of internal rotation with an average of 13 (5) deg and 29 (11) deg, respectively. The PO turning had the largest magnitude in the external direction with -9 (4) deg of displacement (Table 2). Analyzing the activities by TRA setting, the displacement between the socket and residual limb did generally increase with increased TRA stiffness (Table 3). However, significant differences were only found for external rotation between the compliant and intermediate settings and the compliant and stiff settings for PI and PO turning, respectively.

The stiffness of the combined limb-socket body was different between the individual subjects and activities in both the internal and external directions (all $P_{\mathrm{s}}<.001$; Figure 4, Table 4). There were no differences between the 3 walking speeds $(P=.286$ and $P=.301$ for internal and external rotation, respectively). However, there were differences between the fast and slow speeds during 


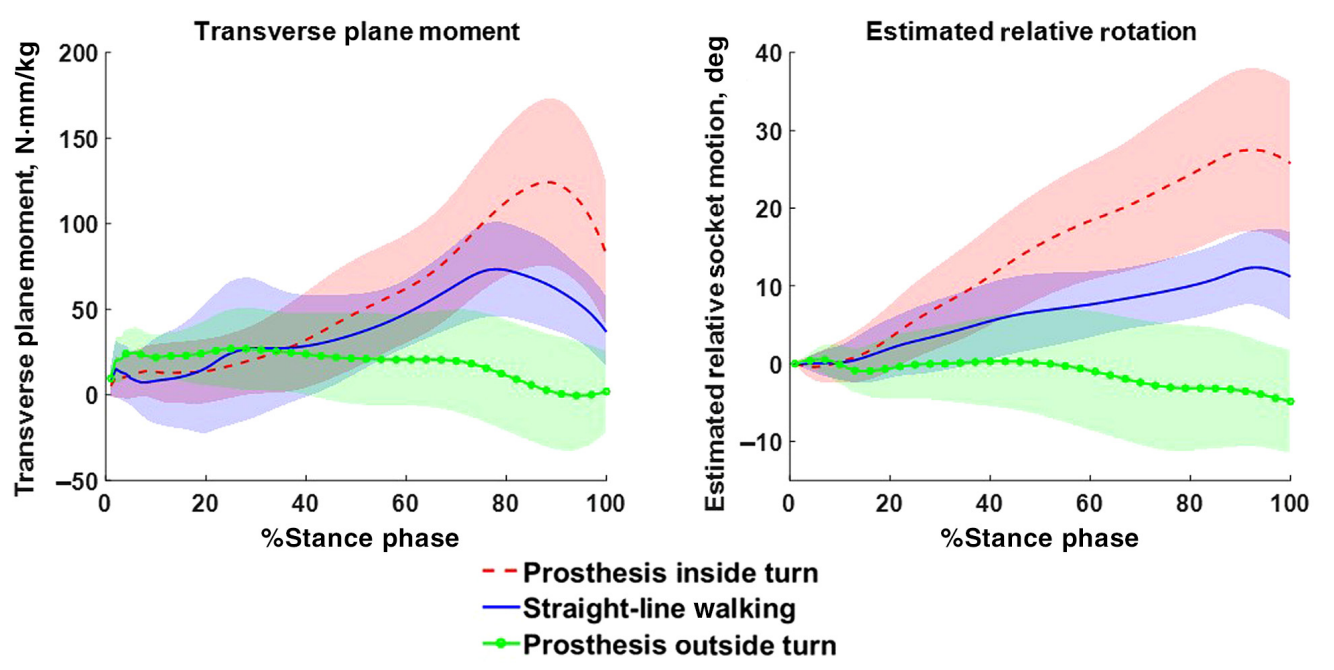

Figure 2 - Mean (bold lines) and SD (shaded regions) for the transverse plane moment measured at the bottom of the socket (left) and the estimated relative motion between the socket and residual limb (right) for the 3 different activities. The horizontal axes show the percent of stance from heel strike to toe-off. These data represent all tested TRA stiffness settings combined. TRA indicates transverse rotation adapter.

(A)

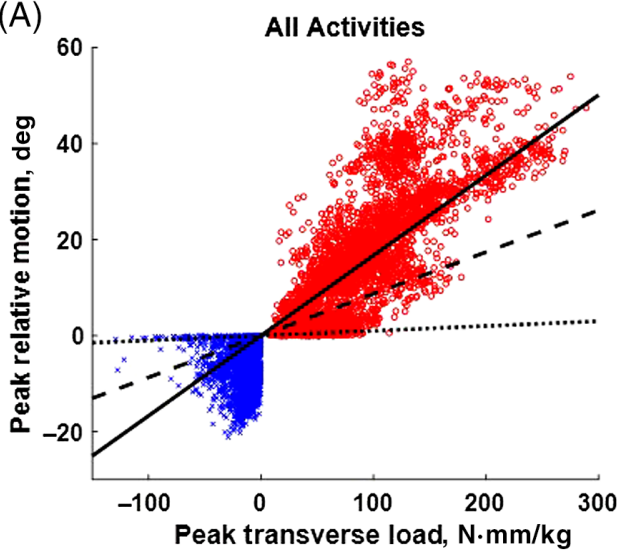

(C)

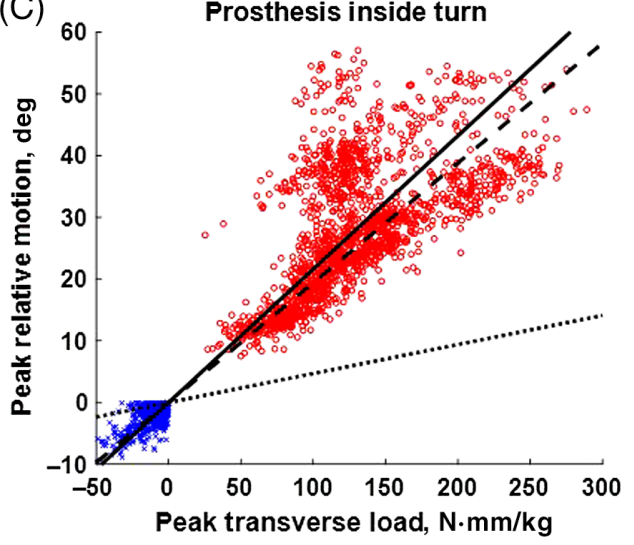

(B) Straight-Line Walking

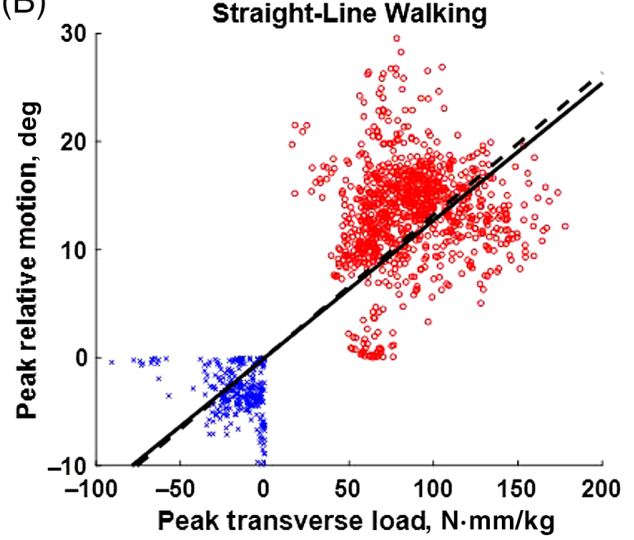

(D) 30 - Prosthesis outside turn

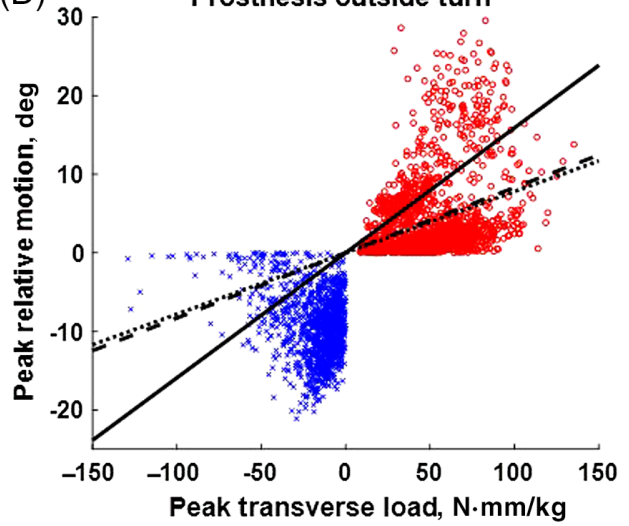

- Internal rotation peaks

$\times \quad$ External rotation peaks

- - - Internal rotation linear fit

........... External rotation linear fit

\section{Combined linear fit}

Figure 3 - Relationship between the peak estimated relative motion between the socket and residual limb ( $y$-axis) and the peak transverse plane moment measured at the bottom of the socket ( $x$-axis). Lines represent the fit from linear regression models. (A) All activities combined, (B) straight-line walking, (C) prosthesis inside turning, and (D) prosthesis outside turning. 
Table 2 Relationship Between the Estimated Peak Transverse Motion Between the Socket and Tibia Segment of the Residual Limb and the Peak TPM Measured at the Distal End of the Socket

\begin{tabular}{|c|c|c|c|c|c|c|}
\hline \multicolumn{7}{|c|}{ Relationship between peak relative motion and peak transverse loading } \\
\hline Activity & Direction & Linear fit, deg/N.mm/kg & $\begin{array}{l}P \text { value } \\
\text { linear fit }\end{array}$ & $\begin{array}{l}P \text { value internal } \\
\text { vs external }\end{array}$ & Peak TPM, N.mm/kg & $\begin{array}{l}\text { Peak estimated } \\
\text { rotation, deg }\end{array}$ \\
\hline \multirow[t]{3}{*}{ All } & All & $0.167(0.001)$ & $<.001$ & $<.001$ & & \\
\hline & Internal & $0.087(0.002)$ & $<.001$ & & $90(49)$ & $16(13)$ \\
\hline & External & $0.010(0.004)$ & .030 & & $-16(15)$ & $-6(5)$ \\
\hline \multirow[t]{3}{*}{ ST } & All & $0.127(0.002)$ & $<.001$ & .005 & & \\
\hline & Internal & $0.132(0.004)$ & $<.001$ & & $87(28)$ & $13(5)$ \\
\hline & External & $0.005(0.004)$ & .189 & & $-16(16)$ & $-3(2)$ \\
\hline \multirow[t]{3}{*}{ PI } & All & $0.216(0.002)$ & $<.001$ & .001 & & \\
\hline & Internal & $0.194(0.004)$ & $<.001$ & & $130(47)$ & $29(11)$ \\
\hline & External & $0.047(0.006)$ & $<.001$ & & $-11(10)$ & $-2(2)$ \\
\hline \multirow[t]{3}{*}{$\mathrm{PO}$} & All & $0.159(0.002)$ & .001 & $<.001$ & & \\
\hline & Internal & $0.083(0.004)$ & $<.001$ & & $51(21)$ & $4(6)$ \\
\hline & External & $0.078(0.006)$ & $<.001$ & & $-18(17)$ & $-9(4)$ \\
\hline
\end{tabular}

Abbreviations: PI, turning with prosthesis on the inside of the turn; PO, turning with prosthesis on the outside of the turn; ST, straight-line walking; TPM, transverse plane moment; TRA, transverse rotation adapter. Note: Linear fit gives the slope of the relationship between the estimated motion and measure moment values (mean [SE]). Peak TPM and peak estimated rotation (mean [SD]) are the average peak values across all individuals, walking speeds, and TRA settings. Significance of the linear fit as well as differences between internal and external rotation slopes $(P<.05)$ are shown in bold.

Table 3 Estimated Peak Transverse Motion Between the Socket and Tibia Segment of the Residual Limb (Mean [SE])

Peak estimated rotation by TRA setting

Comparison of stiffness settings, independent of walking speed

Estimated transverse deflection,

deg

\begin{tabular}{|c|c|c|c|c|c|c|c|}
\hline \multirow[b]{2}{*}{ Activity } & \multirow[b]{2}{*}{ Direction } & \multicolumn{3}{|c|}{ deg } & \multicolumn{3}{|c|}{ Pairwise comparisons ( $P$ value) } \\
\hline & & Compliant & Intermediate & Stiff & Compliant vs intermediate & Compliant vs stiff & Intermediate vs stiff \\
\hline \multirow[t]{2}{*}{ ST } & Internal & $12.8(1.7)$ & $12.9(1.7)$ & $13.0(1.7)$ & 0.890 & 0.371 & 0.717 \\
\hline & External & $-1.7(0.7)$ & $-1.9(0.7)$ & $-1.8(0.7)$ & 0.391 & 0.623 & 0.849 \\
\hline \multirow[t]{2}{*}{ PI } & Internal & $30.9(3.3)$ & $31.2(3.3)$ & $31.3(3.3)$ & 0.375 & 0.137 & 0.923 \\
\hline & External & $-1.4(0.4)$ & $-1.6(0.4)$ & $-1.5(0.4)$ & 0.044 & 0.637 & 0.252 \\
\hline \multirow[t]{2}{*}{$\mathrm{PO}$} & Internal & $5.3(1.8)$ & $5.2(1.8)$ & $5.4(1.8)$ & 0.877 & 0.766 & 0.525 \\
\hline & External & $-6.6(1.4)$ & $-6.8(1.4)$ & $-7.1(1.4)$ & 0.652 & 0.011 & 0.225 \\
\hline
\end{tabular}

Abbreviations: PI, turning with prosthesis on the inside of the turn; PO, turning with prosthesis on the outside of the turn; ST, straight-line walking; TRA, transverse rotation adapter. Note: Estimated deflection in degrees is given for the 3 tested TRA settings (compliant $0.25 \mathrm{Nm} /{ }^{\circ}$, intermediate: $0.75 \mathrm{Nm} /{ }^{\circ}$, and stiff: $1.25 \mathrm{Nm} /{ }^{\circ}$ ) during the 3 activities (ST, PI, and PO). Internal rotation was positive. Pairwise differences between TRA settings are given with significant differences $(P<.05)$ shown in bold.

internal rotation for all 3 activities individually (all $P$ s $<.001)$ and during external rotation for all activities combined and while straight-line walking (all $P_{\mathrm{s}} \leq .008$ ) (Table 5).

\section{Discussion}

The objective of this study was to relate the relative rotational motion between the residual limb and socket that causes shear stresses in the soft tissues with the observed transverse plane load applied to the distal end of the socket using an IK analysis. In addition, the experimentally measured load and estimated displacement were used to calculate the rotational stiffness of the combined socket-limb body for each individual during the different activity and speed combinations.
Our first hypothesis, that the peak transverse plane moment will have a positive, linear relationship to the estimated relative motion between the socket and residual limb, was supported for all activities individually and combined. There was a linear relationship between peak rotational loading at the distal end of the socket and peak estimated rotation between the socket and tibia of the residual limb. While significant relationships were found for each direction individually (Table 2), the estimated slopes did not always align well with the data (eg, external rotation, Figure 3A, 3C and 3D). The linear model accounts for the effects of TRA setting, activity, speed, and variability between individuals. With all the individual subject data being combined in Figure 3, it is difficult to distinguish trends based on visual inspection. The appearance of a poor fit is a result of the limited range of the external data alone when compared with the 


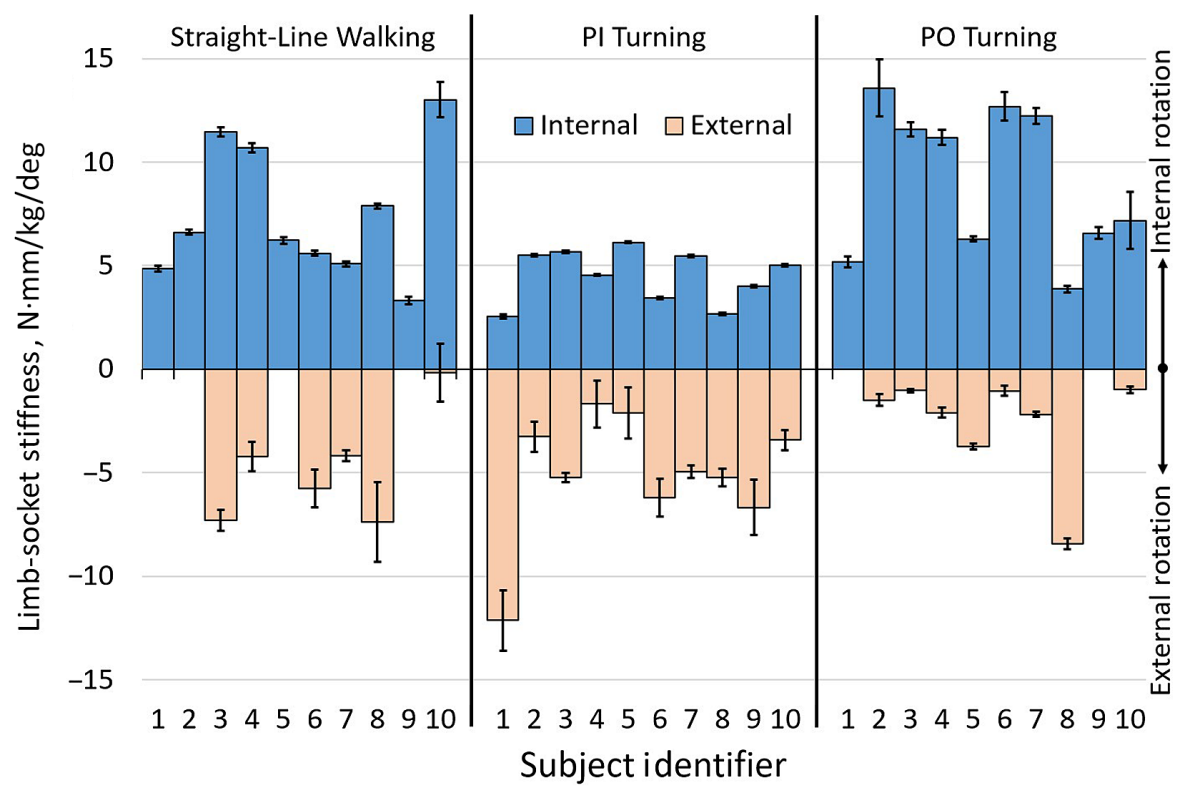

Figure 4 - Limb-socket stiffness for each individual and activity; bars are mean with SE. Individuals without values for external stiffness did not have enough data in the external direction to calculate a value. Subject identifiers correlate to subject numbers in Table 1. PI indicates prosthesis on the inside of the turn; PO, prosthesis on the outside of the turn.

Table 4 Pairwise Comparison Results for LimbSocket Stiffness (Mean [SE]) Between the 3 Activities

\begin{tabular}{lcccc}
\hline \multicolumn{4}{c}{ Transverse socket-limb stiffness comparisons by activity } \\
\hline \multirow{2}{*}{ Direction } & Activity & $\begin{array}{c}\text { Stiffness, } \\
\text { N.mm/kg/deg }\end{array}$ & $\begin{array}{c}\text { Pairwise } \\
\text { comparison }\end{array}$ & $\boldsymbol{P}$ value \\
\hline Internal & ST & $6.80(0.07)$ & ST-PN & $<.001$ \\
& PI & $4.47(0.05)$ & ST-PO & 1.000 \\
& PO & $6.79(0.09)$ & PI-PO & $<.001$ \\
External & ST & $5.72(0.20)$ & ST-PI & .987 \\
& PI & $5.72(0.20)$ & ST-PO & $<.001$ \\
& PO & $2.99(0.28)$ & PI-PO & $<.001$ \\
\hline
\end{tabular}

Abbreviations: PI, turning with prosthesis on the inside of the turn; PO, turning with prosthesis on the outside of the turn; ST, straight-line walking. Note: Significant differences $(P<.05)$ are shown in bold.

internal data, indicating that both internal and external rotation should be combined when estimating the overall transverse plane rotational stiffness of the limb. The significance found in this model is best interpreted that a linear relationship is valid for a given individual under the conditions of TRA setting, activity, and speed. The variance in data highlights how responses differ across individuals, indicating the need for individual-specific TRA setup.

During ST and PI activities, the limb experiences primarily internal rotation. The magnitudes of rotation were found to be 13 (5) deg and 29 (11) deg for straight-line walking and PI turning, respectively. Previous studies have shown relative motion between the socket and residual limb between 3 and $15 \operatorname{deg}^{5,16}$ during straight-line walking, which is consistent with our findings. These studies looked primarily at motion between the socket and liner, which suggests that during ST walking activities, the primary motion may be between the socket and liner with less deformation of the soft tissues. When PI turning, we found that the residual limb rotation is more than double that of straight-line walking. In addition, even within our small sample size, the variation of rotation during ST was large, with some individuals experiencing deflections as high as 30 deg during straight-line walking and up to $60 \mathrm{deg}$ when PI turning. This suggests there may be a large magnitude of deflection in the soft tissues of the residual limb, particularly when turning, which is known to result in deep tissue injury. ${ }^{2,38}$ In addition to skin ailments that are frequently cited for individuals with lower-limb amputation, increased shear stress on the residual limb could occlude blood flow, which could further exacerbate symptoms of pain and discomfort and cause medical complications, particularly for amputees of vascular etiology. ${ }^{20}$

Results from the PO turning were less straightforward. The PO turning method is similar to a cutting maneuver, but less aggressive. This exerted less pure rotation on the limb as compared with straight-line walking and PI turning, which affects the motion of the residual limb seen during PO turns. For straight-line walking and PI turns, the peak internal transverse plane moment corresponds well to the peak estimated rotations (Table 2). However, for PO turns, the limb experiences relatively high loading internally (50 [21] $\mathrm{N} \cdot \mathrm{mm} / \mathrm{kg}$ ) with minimal deflection (4 [6] deg) and relatively low external loading $(-22[20] \mathrm{N} \cdot \mathrm{mm} / \mathrm{kg}$ ) for a larger magnitude of deflection ( -9 [4] deg). As compared with straightline walking and PI turning, during PO turning, the individual pushes laterally at heel strike to initiate the change in direction. This lateral loading induces less internal rotation and includes off-axis loading, which may cause the limb to compress and exhibit greater rotational stiffness, particularly during internal rotation (Table 4). During toe-off, the foot is used to propel the individual out of the turn and into external rotation with a reduced magnitude of rotational stiffness. In addition, the technique used by each individual varied greatly, resulting in a high degree of variance (Figure 3D). Note the high SD for the peak internal loading (peak TPM) and peak rotation (peak estimated rotation) as compared with the mean (Table 2).

In our previous work, we established that increasing the rotational stiffness of the TRA device resulted in significant increases to 
Table 5 Pairwise Comparison Results for Limb-Socket Stiffness (Mean [SE]) Between the 3 Walking Speeds

\begin{tabular}{|c|c|c|c|c|c|}
\hline \multicolumn{6}{|c|}{ Transverse socket-limb stiffness comparisons by speed } \\
\hline Activity & Comparison & Speed & Stiffness, $\mathrm{N} \cdot \mathrm{mm} / \mathrm{kg} / \mathrm{deg}$ & Pairwise comparison & $P$ value \\
\hline \multirow[t]{6}{*}{ All } & Internal & Fast & $5.99(0.07)$ & Fast - Slow & .405 \\
\hline & & SSW & $5.96(0.07)$ & Fast - SSW & .914 \\
\hline & & Slow & $6.11(0.07)$ & Slow - SSW & .196 \\
\hline & External & Fast & $5.06(0.21)$ & Fast - Slow & .008 \\
\hline & & SSW & $4.84(0.22)$ & Fast - SSW & .491 \\
\hline & & Slow & $4.50(0.22)$ & Slow - SSW & .143 \\
\hline \multirow[t]{6}{*}{ ST } & Internal & Fast & $7.21(0.12)$ & Fast - Slow & $<.001$ \\
\hline & & SSW & $7.35(0.13)$ & Fast - SSW & .527 \\
\hline & & Slow & $7.86(0.13)$ & Slow - SSW & $<.001$ \\
\hline & External & Fast & $6.28(0.57)$ & Fast - Slow & $<.001$ \\
\hline & & SSW & $5.08(0.53)$ & Fast - SSW & .076 \\
\hline & & Slow & $3.16(0.53)$ & Slow - SSW & $<.001$ \\
\hline \multirow[t]{6}{*}{ PI } & Internal & Fast & $4.47(0.03)$ & Fast - Slow & $<.001$ \\
\hline & & SSW & $4.38(0.03)$ & Fast - SSW & .083 \\
\hline & & Slow & $4.65(0.03)$ & Slow - SSW & $<.001$ \\
\hline & External & Fast & $5.18(0.35)$ & Fast - Slow & .376 \\
\hline & & SSW & $5.41(0.37)$ & Fast - SSW & .809 \\
\hline & & Slow & $4.68(0.39)$ & Slow - SSW & .137 \\
\hline \multirow[t]{6}{*}{ PO } & Internal & Fast & $9.49(0.25)$ & Fast - Slow & $<.001$ \\
\hline & & SSW & $8.89(0.25)$ & Fast - SSW & .009 \\
\hline & & Slow & $8.72(0.25)$ & Slow - SSW & .677 \\
\hline & External & Fast & $2.59(0.11)$ & Fast - Slow & .936 \\
\hline & & SSW & $2.65(0.10)$ & Fast - SSW & .893 \\
\hline & & Slow & $2.63(0.10)$ & Slow - SSW & .991 \\
\hline
\end{tabular}

Abbreviations: PI, turning with prosthesis on the inside of the turn; PO, turning with prosthesis on the outside of the turn; SSW, self-selected walking speed; ST, straight-line walking. Note: Walking speeds are defined as SSW, slow, and fast. Significant differences $(P<.05)$ are shown in bold.

limb loading. ${ }^{14}$ In the present study, we found that a significant, linear relationship does exist between limb loading and estimated displacement (Table 2). However, a significant relationship between TRA setting and displacement is generally not supported (Table 3), although there is a general trend of increased TRA stiffness resulting in increased rotation. This result is consistent with previous work. ${ }^{14}$ Specifically, similar to the relationships we found between TRA setting and limb loading, PI turning experiences the greatest magnitude of displacement overall, and straight-line walking and PI turning experience primarily internal rotation while PO turning generally experiences external rotation. The lack of significant differences between the estimated displacements and TRA settings may indicate a reduced resolution for the model to predict displacement between the socket and limb as compared with direct measurement of limb loading. This would suggest that it may be difficult to use this modeling method to detect small changes in the user interface due to changes in prosthesis setup.

Our second hypothesis, that the transverse stiffness of the residual limb-socket body will vary based on each individual subject, activity, and speed, was also supported. We found that the rotational stiffness was unique to each individual and varied based on activity and speed. The rotational stiffness of the limbsocket body is an important factor when determining the setup of the TRA. Traditionally, the TRA is set based on a person's weight, ambulatory ability, and the experience of the prosthetist fitting the device. However, given the individual-specific information from this method, the lower limb could be modeled as a mechanical system, as has been done previously, ${ }^{26-31}$ with the limb-socket body and TRA represented as 2 springs in a series. Future work should focus on developing a model that would provide guidelines to inform the prescription and setup of a TRA device using this individual-specific information. Lastly, the change in limb-socket body stiffness based on activity and speed would suggest the need for a dynamic TRA that can adjust its stiffness to meet the changing activities of daily living, a conclusion consistent with our previous work. ${ }^{12,14}$

Using this method to evaluate the rotation of the residual limb inside of the socket and estimate the stiffness of an individual's combined limb-socket body could be valuable in clinical practice. Clinical motion capture is effectively used to evaluate an individual's walking mechanics for use in treatment. ${ }^{39}$ This method could be used to provide a greater understanding to clinicians regarding the fit and setup of an individual's socket and prosthesis. Using a minimal marker set to track the lower-limb segments, the rotation of the residual limb could be evaluated to determine if the socket fit is adequate based on the amount of rotation in the residual limb in addition to informing the setup of prosthetic equipment like a TRA. This would help optimize the prosthesis for the individual with a more consistent method of evaluation, which would improve individual care and reduce the time to fit a prosthetic device.

This work was performed to better understand the dynamic response of the residual limb inside the socket of a transtibial amputee; however, some limitations should be noted. These data 
are from a relatively small sample size $(\mathrm{n}=10)$, mostly male, traumatic, transtibial amputees. While statistical significance was found for some outcome metrics, more subjects would be needed to apply these results to a general amputee population, including amputees of diabetic/dysvascular etiology as well as transfemoral amputees. In addition, the time since amputation varies widely between individuals and, as such, the maturity of their residual limb and the properties of the tissues should be investigated in future studies as an interacting factor. Furthermore, the values for limb displacement represent an overall estimate based on the IK analysis, and the limb stiffness is based on that estimate. Currently, we are unable to distinguish between surface motions that may be more responsible for skin abrasions, lesions, and cysts as compared with deeper tissue displacements. Future work should be done to experimentally measure these displacements independently in order to improve reliability and establish validity. We also note that this work focuses solely on the rotational stress applied to the limb, which is only a single component of the principle stress experienced by the soft tissues. Future work should look at additional stresses (compression and linear displacement) experienced by the soft tissues of the limb to better understand all the displacements that may affect tissue health and ultimately improve the fitment of prosthetic devices. Finally, we acknowledge that the use of fixed TRA settings across all individuals is not ideal and have addressed this in our previous work. ${ }^{14}$

In summary, this study found that there was a significant relationship between peak transverse loading measured at the distal end of the socket and peak estimated displacement between the socket and inner residual limb that result in soft-tissue shear stress. These findings suggest that measuring the rotational load at the socket is an acceptable method to indicate rotational soft-tissue shear of the residual limb during experimental trials and that the use of an IK analysis may be a valid approach to measure displacement between the socket and limb. In addition, we found that the rotational stiffness of the combined limb-socket body varied significantly between individuals, activity type, and speed. Variations in stiffness suggest that the coupling between the socket and limb is a characteristic unique to each individual and one that varies dynamically when performing different activities and at different speeds. This means that the setup of a device like a TRA might be better informed by a clinical analysis of limb-socket stiffness to meet the needs of each individual and that it should have the ability to dynamically adapt to changing activities and speeds.

\section{Acknowledgments}

This work was supported by awards I21 RX001933, IK6 RX002974, and I50 RX002357 from the US Department of Veterans Affairs Rehabilitation Research and Development Service. The contents do not represent the views of the US Department of Veterans Affairs or the US Government. The authors have no conflicts of interest to disclose.

\section{References}

1. Sanders JE, Daly CH, Burgess EM. Interface shear stresses during ambulation with a below-knee prosthetic limb. J Rehabil Res Dev. 1992;29(4):1-8. PubMed ID: 1432723 doi:10.1682/JRRD.1992.10. 0001

2. Portnoy S, Yizhar Z, Shabshin N, et al. Internal mechanical conditions in the soft tissues of a residual limb of a trans-tibial amputee.
J Biomech. 2008;41(9):1897-1909. PubMed ID: 18495134 doi:10. 1016/j.jbiomech.2008.03.035

3. Twiste M, Rithalia S. Transverse rotation and longitudinal translation during prosthetic gait-a literature review. J Rehabil Res Dev. 2003; 40(1):9-18. PubMed ID: 15150716 doi:10.1682/JRRD.2003.01.0009

4. Flick KC, Orendurff MS, Berge JS, Segal AD, Klute GK. Comparison of human turning gait with the mechanical performance of lower limb prosthetic transverse rotation adapters. Prosthet Orthot Int. 2005;29(1):73-81. PubMed ID: 16180379 doi:10.1080/0309364 0500088120

5. Lamoureux LW, Radcliffe CW. Functional analysis of the UC-BL shank axial rotation device. Prosthet Orthot Int. 1977;1(2):114-118. PubMed ID: 615294 doi:10.3109/03093647709164619

6. Heitzmann DWW, Pieschel K, Alimusaj M, Block J, Putz C, Wolf SI. Functional effects of a prosthetic torsion adapter in trans-tibial amputees during unplanned spin and step turns. Prosthet Orthot Int. 2016;40(5):558-565. PubMed ID: 26195621 doi:10.1177/ 0309364615592698

7. Segal AD, Orendurff MS, Czerniecki JM, Shofer JB, Klute GK. Transtibial amputee joint rotation moments during straight-line walking and a common turning task with and without a torsion adapter. J Rehabil Res Dev. 2009;46(3):375-383. PubMed ID: 19675989 doi:10.1682/JRRD.2008.06.0070

8. Buckley JG, Jones SF, Birch KM. Oxygen consumption during ambulation: comparison of using a prosthesis fitted with and without a tele-torsion device. Arch Phys Med Rehabil. 2002;83(4):576-581. PubMed ID: 11932866 doi:10.1053/apmr.2002.30624

9. Su P-F, Gard SA, Lipshutz RD, Kuiken TA. The effects of increased prosthetic ankle motions on the gait of persons with bilateral transtibial amputations. Am J Phys Med Rehabil. 2010;89(1):34-47. PubMed ID: 20026945 doi:10.1097/PHM.0b013e3181c55ad4

10. Segal AD, Kracht R, Klute GK. Does a torsion adapter improve functional mobility, pain, and fatigue in patients with transtibial amputation? Clin Orthop Relat Res. 2014;472(10):3085-3092. PubMed ID: 24733445 doi:10.1007/s11999-014-3607-9

11. Pew C, Klute GK. Design of lower limb prosthesis transverse plane adaptor with variable stiffness. J Med Device. 2015;9(3):035001. doi:10.1115/1.4030505

12. Pew C, Klute GK. Pilot testing of a variable stiffness transverse plane adapter for lower limb amputees. Gait Posture. 2017;51:104-108. PubMed ID: 27744248 doi:10.1016/j.gaitpost.2016.10.003

13. Pew C, Klute GK. Second generation prototype of a variable stiffness transverse plane adapter of a lower limb prosthesis. Med Eng Phys. 2017;49:22-27. PubMed ID: 28807513 doi:10.1016/j.medengphy. 2017.07.002

14. Pew C, Segal AD, Neptune RR, Klute GK. Ideal operating conditions for a variable stiffness transverse plane adapter for individuals with lower-limb amputation. J Biomech. 2019;96(11):109330. doi:10. 1016/j.jbiomech.2019.109330

15. LaPrè AK, Price MA, Wedge RD, Umberger BR, Sup FC. Approach for gait analysis in persons with limb loss including residuum and prosthesis socket dynamics. Int J Numer Method Biomed Eng. 2018; 34(4):1-11. doi:10.1002/cnm.2936

16. Childers WL, Siebert S. Marker-based method to measure movement between the residual limb and a transtibial prosthetic socket. Prosthet Orthot Int. 2016;40(6):720-728. PubMed ID: 26527758 doi:10.1177/ 0309364615610660

17. Gholizadeh H, Osman NAA, Kamyab M, Eshraghi A, Abas WABW, Azam MN. Transtibial prosthetic socket pistoning: static evaluation of Seal-In ${ }^{\circledR}$ X5 and Dermo ${ }^{\circledR}$ Liner using motion analysis system. Clin Biomech. 2012;27(1):34-39. doi:10.1016/j.clinbiomech.2011. 07.004 
18. Lenz AL, Johnson KA, Bush TR. A new method to quantify liner deformation within a prosthetic socket for below knee amputees. $J$ Biomech. 2018;74:213-219. PubMed ID: 29678418 doi:10.1016/j. jbiomech.2018.04.008

19. Zhang M, Lord M, Turner-Smith AR, Roberts VC. Development of a non-linear finite element modelling of the below-knee prosthetic socket interface. Med Eng Phys. 1995;17(8):559-566. PubMed ID: 8564149 doi:10.1016/1350-4533(95)00002-5

20. Linder-Ganz E, Gefen A. The effects of pressure and shear on capillary closure in the microstructure of skeletal muscles. Ann Biomed Eng. 2007;35(12):2095-2107. doi:10.1007/s10439-007-9384-9

21. Convery P, Murray KD. Ultrasound study of the motion of the residual femur within a trans-femoral socket during daily living activities other than gait. Prosthet Orthot Int. 2000;24(3):226-232. PubMed ID: 11195358 doi:10.1080/03093640008726552

22. Commean PK, Smith KE, Vannier MW. Lower extremity residual limb slippage within the prosthesis. Arch Phys Med Rehabil. 1997;78(5): 476-485. PubMed ID: 9161365 doi:10.1016/S0003-9993(97)90160-X

23. Chong SY, Röhrle O. Exploring the use of non-image-based ultrasound to detect the position of the residual femur within a stump. PLoS One. 2016;11(10):1-22. doi:10.1371/journal.pone.0164583

24. Grevsten S, Erikson U. A roentgenological study of the stump-socket contact and skeletal displacement in the PTB-suction prosthesis. Ups J Med Sci. 1975;80(1):49-57. PubMed ID: 1145905 doi:10.3109/ 03009737509178991

25. Lilja M, Johansson T, Öberg T. Movement of the tibial end in a PTB prosthesis socket: a sagittal X-ray study of the PTB prosthesis. Prosthet Orthot Int. 1993;17(1):21-26. PubMed ID: 8337097 doi:10. 3109/03093649309164351

26. Glaister BC, Schoen JA, Orendurff MS, Klute GK. Mechanical behavior of the human ankle in the transverse plane while turning. IEEE Trans Neural Syst Rehabil Eng. 2007;15(4):552-559. PubMed ID: 18198713 doi:10.1109/TNSRE.2007.908944

27. Glaister BC, Schoen JA, Orendurff MS, Klute GK. A mechanical model of the human ankle in the transverse plane during straight walking: implications for prosthetic design. J Biomech Eng. 2009; 131(3):034501. PubMed ID: 19154072 doi:10.1115/1.3005153

28. Rouse EJ, Gregg RD, Hargrove LJ, Sensinger JW. The difference between stiffness and quasi-stiffness in the context of biomechanical modeling. IEEE Trans Biomed Eng. 2013;60(2):562-568. PubMed ID: 23212310 doi:10.1109/TBME.2012.2230261
29. Hedrick EA, Stanhope SJ, Takahashi KZ. The foot and ankle structures reveal emergent properties analogous to passive springs during human walking. PLoS One. 2019;14(6):e0218047. doi:10. 1371/journal.pone.0218047

30. Klute GK, Kallfelz CF, Czerniecki JM, Czerniecki MJM. Mechanical properties of prosthetic limbs: adapting to the patient. J Rehabil Res Dev. 2001;38(3):299-307. PubMed ID: 11440261

31. Sensinger JW, Weir RF. Modeling and preliminary testing socketresidual limb interface stiffness of above-elbow prostheses. IEEE Trans Neural Syst Rehabil Eng. 2008;16(2):184-190. PubMed ID: 18403287 doi:10.1109/TNSRE.2008.918388

32. Lee WCC, Zhang M, Jia X, Cheung JTM. Finite element modeling of the contact interface between trans-tibial residual limb and prosthetic socket. Med Eng Phys. 2004;26(8):655-662. PubMed ID: 15471693 doi:10.1016/j.medengphy.2004.04.010

33. Cagle JC, Reinhall PG, Allyn KJ, et al. A finite element model to assess transtibial prosthetic sockets with elastomeric liners. Med Biol Eng Comput. 2018;56(7):1227-1240. PubMed ID: 29235055 doi:10. 1007/s11517-017-1758-z

34. Tang J, McGrath M, Laszczak P, et al. Characterisation of dynamic couplings at lower limb residuum/socket interface using 3D motion capture. Med Eng Phys. 2015;37(12):1162-1168. PubMed ID: 26525779 doi:10.1016/j.medengphy.2015.10.004

35. Segal AD, Orendurff MS, Czerniecki JM, Schoen J, Klute GK. Comparison of transtibial amputee and non-amputee biomechanics during a common turning task. Gait Posture. 2011;33(1):41-47. PubMed ID: 20974535 doi:10.1016/j.gaitpost.2010.09.021

36. Delp SL, Anderson FC, Arnold AS, et al. OpenSim: open source to create and analyze dynamic simulations of movement. IEEE Trans Biomed Eng. 2007;54(11):1940-1950. PubMed ID: 18018689 doi:10. 1109/TBME.2007.901024

37. Hicks JL, Uchida TK, Seth A, Rajagopal A, Delp SL. Is my model good enough? Best practices for verification and validation of musculoskeletal models and simulations of movement. J Biomech Eng. 2015;137(2):020905. doi:10.1115/1.4029304

38. Gefen A. Risk factors for a pressure-related deep tissue injury: a theoretical model. Med Biol Eng Comput. 2007;45(6):563-573. PubMed ID: 17486382 doi:10.1007/s11517-007-0187-9

39. Wren TAL, Gorton GE, Õunpuu S, Tucker CA. Efficacy of clinical gait analysis: a systematic review. Gait Posture. 2011;34(2):149153. PubMed ID: 21646022 doi:10.1016/j.gaitpost.2011.03.027 